\title{
Global stability of a fractional order HIV infection model with cure of infected cells in eclipse stage
}

\author{
Moussa Bachraoui ${ }^{a}$, Khalid Hattaf ${ }^{a, b}$, Noura Yousfi ${ }^{a}$ \\ ${ }^{a}$ Laboratory of Analysis, Modeling and Simulation (LAMS), Faculty of Sciences Ben M'sik, \\ Hassan II University, P.O Box 7955 Sidi Othman, Casablanca, Morocco \\ ${ }^{b}$ Centre Régional des Métiers de l'Education et de la Formation (CRMEF), \\ 20340 Derb Ghalef, Casablanca, Morocco
}

\begin{abstract}
Modeling by fractional order differential equations has more advantages to describe the dynamics of phenomena with memory which exists in many biological systems. In this paper, we propose a fractional order model for human immunodeficiency virus (HIV) infection by including a class of infected cells that are not yet producing virus, i.e., cells in the eclipse stage. We first prove the positivity and boundedness of solutions in order to ensure the well-posedness of the proposed model. By constructing appropriate Lyapunov functionals, the global stability of the disease-free equilibrium and the chronic infection equilibrium is established. Numerical simulations are presented in order to validate our theoretical results.
\end{abstract}

Keywords: HIV infection, eclipse stage, nonlinear incidence rate, global stability.

\section{Introduction}

In recent years, many mathematical models used fractional order differential equations (FDEs) have been developed to better describe the dynamics of viral infections such as the human immunodeficiency virus (HIV), the hepatitis $\mathrm{B}$ virus (HBV) and the hepatitis $\mathrm{C}$ virus (HCV). In 2012, Arafa et al. [1] introduced fractional-order into a model of HIV infection of $\mathrm{CD} 4^{+} \mathrm{T}$ cells and they studied the effect of the changing the average number of viral particles with different sets of initial conditions on the dynamics of the presented model. In 2016, Liu et al. [2] proposed a fractional mathematical model which 
includes cure rate and Beddington-DeAngelis functional response. They established only the local stability of equilibria, but not investigated the global stability of these equilibria. In 2017, Salman and Yousef [3] considered a fractional-order model for HBV infection with cure of infected cells and they discussed the local asymptotic stability of equilibria. In the same years, Boukhouima et al. [4] generalized all the above models by modeling the infection transmission process by Hattaf's incidence rate [5]. This incidence rate was used by many authors [6-9] and it covers many common types existing in the literature, such as the bilinear incidence function called also the mass action, the saturation incidence rate, the Beddington-DeAnglis functional response $[10,11]$ and the Crowley-Martin functional response [12]. In the above fractional-order models [1-4], infected cells are assumed to produce new virions immediately after target cells are infected by a free virus. However, there are many biological steps between viral infection of target cells and the production of new virions. In our study, we extend and improve these fractional models by incorporating an eclipse phase, representing the stage in which infected cells have not started to produce new virions.

The rest of this paper is outlined as follows. In the next section, we formulate our fractional model and give their basic properties. In Section 3, by constructing suitable Lyapunov functionals, the global stability of equilibria is investigated. Numerical simulations are presented in Section 4. Finally, we conclude our results and give future work.

\section{Model formulation and basic properties}

The first aim of this paper is to extend and improve the fractional-order models [1-4] by proposing the following model

$$
\begin{aligned}
& D^{\alpha} T(t)=\lambda-\mu_{T} T(t)-f(T(t), V(t)) V(t)+\rho E(t), \\
& D^{\alpha} E(t)=f(T(t), V(t)) V(t)-\left(\mu_{E}+\rho+\gamma\right) E(t), \\
& D^{\alpha} I(t)=\gamma E(t)-\mu_{I} I(t) \\
& D^{\alpha} V(t)=k I(t)-\mu_{V} V(t),
\end{aligned}
$$

where $D^{\alpha}$ is fractional derivative in the Caputo sense and $\alpha$ is a parameter that describes the order of the fractional time-derivative with $0<\alpha \leq 1$. The variables $T(t), E(t), I(t), V(t)$ denote the concentrations of uninfected $\mathrm{CD} 4^{+} \mathrm{T}$ cells, infected cells in the eclipse stage (unproductive infected cells), 
productive infected cells and free HIV particles at time $t$, respectively. The constant $\lambda$ is the production rate of infected CD4 ${ }^{+} \mathrm{T}$ cells and $\mu_{T}$ is their natural death rate, and $f(T, V) V=\frac{\beta T V}{1+\alpha_{1} T+\alpha_{2} V+\alpha_{3} T V}$ describes the incidence of $\mathrm{HIV}$ infection of health $\mathrm{CD} 4^{+} \mathrm{T}$ cells, where $\alpha_{1}, \alpha_{2}, \alpha_{3} \geq 0$ are the saturation factors measuring the inhibitory or psychological effect, and $\beta$ is the infection rate. The unproductive infected cells die at the rate $\mu_{E}$, return to the uninfected cells at the rate $\rho$ and become productive infected cells at the rate $\gamma$. Productive infected cells die at the rate $\mu_{I}$. Free HIV particles are produced from infected cells at the rate $k$ and cleared at the rate $\mu_{V}$. It is very important to note when $\alpha=1$, system (1) becomes a model with an ordinary derivative presented by Hattaf and al. in [8] which is the generalization of ODE models presented in $[14,16]$.

The use of fractional derivative in our model is justified by the fact that the membranes of cells of biological organisms have fractional order electrical conductance [17]. Further, the comparisons between the results of the fractional-order model, the results of the integer model and the measured real data obtained from 10 patients during HIV infection show that the results of the fractional-order model give predictions to the plasma virus load of the patients better than those of the integer model [18].

For biological reasons, we assume that the initial data for system (1) satisfy:

$$
T(0)=T_{0} \geq 0, E(0)=E_{0} \geq 0, I(0)=I_{0} \geq 0, V(0)=V_{0} \geq 0 .
$$

First, we have the following result.

Theorem 2.1. For any initial data satisfying (2), system (1) has a unique solution on $[0,+\infty)$. This solution remains non-negative and bounded for all $t \geq 0$. Moreover, we have

(i) $N(t) \leq N(0)+\frac{\lambda}{\delta}$

(ii) $V(t) \leq V(0)+\frac{k}{\mu_{V}}\|I\|_{\infty}$

where $N(t)=T(t)+E(t)+I(t)$ and $\delta=\min \left\{\mu_{T}, \mu_{E}, \mu_{I}\right\}$. 
Proof. From (1), we have

$$
\begin{aligned}
\left.D^{\alpha} T\right|_{T=0} & =\lambda+\rho E \geq 0, \\
\left.D^{\alpha} E\right|_{E=0} & =f(T, V) V \geq 0, \\
\left.D^{\alpha} I\right|_{I=0} & =\gamma E \geq 0, \\
\left.D^{\alpha} V\right|_{V=0} & =k I \geq 0 .
\end{aligned}
$$

It follows from [13] that the set $\mathbb{R}_{+}^{4}=\left\{(T, E, I, V) \in \mathbb{R}^{4}: T \geq 0, E \geq 0, I \geq\right.$ $0, V \geq 0\}$ is positively invariant.

It is not hard to see that the vector function of system (1) satisfies the first condition of Lemma 4 in [4]. It remains to show the second condition condition of this Lemma. Let

$$
X(t)=\left(\begin{array}{c}
T(t) \\
E(t) \\
I(t) \\
V(t)
\end{array}\right) \text { and } \eta=\left(\begin{array}{c}
\lambda \\
0 \\
0 \\
0
\end{array}\right)
$$

So, we discuss four cases:

- If $\alpha_{1} \neq 0$, then system (1) can be written as follows

$$
D^{\alpha} X(t)=\eta+A_{1} X+\frac{\alpha_{1} T}{1+\alpha_{1} T+\alpha_{2} V+\alpha_{3} T V} A_{2} X
$$

where

$$
A_{1}=\left(\begin{array}{cccc}
-\mu_{T} & \rho & 0 & 0 \\
0 & -\left(\mu_{E}+\rho+\gamma\right) & 0 & 0 \\
0 & \gamma & -\mu_{I} & 0 \\
0 & 0 & k & -\mu_{V}
\end{array}\right) \text { and } A_{2}=\left(\begin{array}{cccc}
0 & 0 & 0 & \frac{-\beta}{\alpha_{1}} \\
0 & 0 & 0 & \frac{\beta}{\alpha_{1}} \\
0 & 0 & 0 & 0 \\
0 & 0 & 0 & 0
\end{array}\right)
$$

Then

$$
\left\|D^{\alpha} X(t)\right\| \leq\|\eta\|+\left(\left\|A_{1}\right\|+\left\|A_{2}\right\|\right)\|X\|
$$

- If $\alpha_{2} \neq 0$, we have

$$
D^{\alpha} X(t)=\eta+A_{1} X+\frac{\alpha_{2} V}{1+\alpha_{1} T+\alpha_{2} V+\alpha_{3} T V} A_{3} X
$$


where

$$
A_{3}=\left(\begin{array}{cccc}
\frac{-\beta}{\alpha_{2}} & 0 & 0 & 0 \\
\frac{\beta}{\alpha_{2}} & 0 & 0 & 0 \\
0 & 0 & 0 & 0 \\
0 & 0 & 0 & 0
\end{array}\right)
$$

Then

$$
\left\|D^{\alpha} X(t)\right\| \leq\|\eta\|+\left(\left\|A_{1}\right\|+\left\|A_{3}\right\|\right)\|X\|
$$

- If $\alpha_{3} \neq 0$, we have

$$
D^{\alpha} X(t)=\eta+A_{1} X+\frac{\alpha_{3} T V}{1+\alpha_{1} T+\alpha_{2} V+\alpha_{3} T V} A_{4} X,
$$

where

$$
A_{4}=\left(\begin{array}{cccc}
\frac{-1}{\alpha_{3}} & 0 & 0 & 0 \\
\frac{1}{\alpha_{3}} & 0 & 0 & 0 \\
0 & 0 & 0 & 0 \\
0 & 0 & 0 & 0
\end{array}\right)
$$

Then

$$
\left\|D^{\alpha} X(t)\right\| \leq\|\eta\|+\left(\left\|A_{1}\right\|+\left\|A_{4}\right\|\right)\|X\|
$$

- If $\alpha_{1}=\alpha_{2}=\alpha_{3}=0$, we have

$$
D^{\alpha} X(t)=\zeta+A_{1} X+V A_{5} X
$$

where

$$
A_{5}=\left(\begin{array}{cccc}
-\beta & 0 & 0 & 0 \\
\beta & 0 & 0 & 0 \\
0 & 0 & 0 & 0 \\
0 & 0 & 0 & 0
\end{array}\right)
$$

Then

$$
\left\|D^{\alpha} X(t)\right\| \leq\|\zeta\|+\left(\|V\|\left\|A_{5}\right\|+\left\|A_{1}\right\|\right)\|X\| .
$$

Hence, the second condition of Lemma 4 in [4] is satisfied. Therefore, system (1) has a unique solution on $[0,+\infty)$.

By adding the first three equations of system (1), we obtain

$$
D^{\alpha} N(t) \leq \lambda-\delta N(t)
$$


which implies that

$$
N(t) \leq N(0) E_{\alpha}\left(-\delta t^{\alpha}\right)+\frac{\lambda}{\delta}\left[1-E_{\alpha}\left(-\delta t^{\alpha}\right)\right]
$$

where $E_{\alpha}(z)=\sum_{k=0}^{\infty} \frac{z^{\alpha}}{\Gamma(\alpha k+1)}$ is the Mittag-Leffler function of parameter $\alpha$. Since $0 \leq E_{\alpha}\left(-\delta t^{\alpha}\right) \leq 1$, we deduce (i).

Now, we show (ii). The fourth equation of system (1) implies that

$$
V(t)=V(0) E_{\alpha}\left(-\mu_{V} t^{\alpha}\right)+k \int_{0}^{t} \alpha I(s)(t-s)^{\alpha-1} E_{\alpha}^{\prime}\left(-\mu_{V}(t-s)^{\alpha}\right) d s .
$$

Then

$$
V(t) \leq V(0) E_{\alpha}\left(-\mu_{V} t^{\alpha}\right)+\frac{k}{\mu_{V}}\|I\|_{\infty}\left[1-E_{\alpha}\left(-\mu_{V} t^{\alpha}\right)\right]
$$

Thus,

$$
V(t) \leq V(0)+\frac{k}{\mu_{V}}\|I\|_{\infty}
$$

We begin the analysis of the equilibria by observing that system (1) has a disease-free equilibrium $Q_{0}\left(\frac{\lambda}{\mu_{T}}, 0,0,0\right)$. Then we define the basic reproduction number of (1) as follows

$$
R_{0}=\frac{\lambda \beta k \gamma}{\mu_{I} \mu_{V}\left(\lambda \alpha_{1}+\mu_{T}\right)\left(\rho+\mu_{E}+\gamma\right)}
$$

which represents the average number of secondary infections produced by one productive infected cell during the period of infection when all cells are uninfected.

Similarly to [8], it is not hard to get the following result.

\section{Theorem 2.2.}

(i) If $R_{0} \leq 1$, then the system (1) has a unique disease-free equilibrium of the form $Q_{0}\left(T_{0}, 0,0,0\right)$, where $T_{0}=\frac{\lambda}{\mu_{T}}$. 
(ii) If $R_{0}>1$, the disease-free equilibrium is still present and the system (1) has a unique chronic infection equilibrium of the form $Q_{1}\left(T_{1}, E_{1}, I_{1}, V_{1}\right)$ where $T_{1} \in\left(0, \frac{\lambda}{\mu_{T}}\right), E_{1}=\frac{\lambda-\mu_{T} T_{1}}{\mu_{E}+\gamma}, I_{1}=\frac{\gamma\left(\lambda-\mu_{T} T_{1}\right)}{\mu_{I}\left(\mu_{E}+\gamma\right)}$ and $V_{1}=$ $\frac{k \gamma\left(\lambda-\mu_{T} T_{1}\right)}{\mu_{I} \mu_{V}\left(\mu_{E}+\gamma\right)}$

\section{Global stability}

In this section, we establish the global stability of the disease-free equilibrium $Q_{0}$ and the chronic infection equilibrium $Q_{1}$.

Theorem 3.1. If $R_{0} \leq 1$, then the disease-free equilibrium $Q_{0}$ is globally asymptotically stable, and becomes unstable if $R_{0}>1$.

Proof. Consider the following Lyapunov functional

$$
\begin{aligned}
L_{0}(t)= & \frac{T_{0}}{1+\alpha_{1} T_{0}} \Phi\left(\frac{T}{T_{0}}\right)+\frac{\rho\left(T-T_{0}+E\right)^{2}}{2\left(1+\alpha_{1} T_{0}\right)\left(\mu_{T}+\mu_{E}+\gamma\right) T_{0}} \\
& +\frac{\rho+\mu_{E}+\gamma}{\gamma} I+E+\frac{\mu_{I}\left(\rho+\mu_{E}+\gamma\right)}{k \gamma} V,
\end{aligned}
$$

where $\Phi(x)=x-1-\ln (x), x>0$. By using the property of fractional derivatives given in [19], we can compute

$$
\begin{aligned}
D^{\alpha} L_{0}(t) \leq & \frac{1}{1+\alpha_{1} T_{0}}\left(1-\frac{T_{0}}{T}\right) D^{\alpha} T+\frac{\rho\left(T-T_{0}+E\right)\left(D^{\alpha} T+D^{\alpha} E\right)}{2\left(1+\alpha_{1} T_{0}\right)\left(\mu_{T}+\mu_{E}+\gamma\right) T_{0}} \\
& +\frac{\rho+\mu_{E}+\gamma}{\gamma} D^{\alpha} I+D^{\alpha} E+\frac{\mu_{I}\left(\rho+\mu_{E}+\gamma\right)}{k \gamma} D^{\alpha} V .
\end{aligned}
$$

Using $\lambda=\mu_{T} T_{0}$, we get

$$
\begin{aligned}
D^{\alpha} L_{0}(t) \leq & -\frac{\mu_{T}\left(T_{0}-T\right)^{2}}{\left(1+\alpha_{1} T_{0}\right) T}+\frac{\left(1+\alpha_{1} T\right) T_{0} f(T, V)}{\left(1+\alpha_{1} T_{0}\right) T} V+\rho \frac{\left(T-T_{0}\right) E}{\left(1+\alpha_{1} T_{0}\right) T} \\
& -\frac{\rho \mu_{T}\left(T-T_{0}\right)^{2}}{\left(1+\alpha_{1} T_{0}\right)\left(\mu_{T}+\mu_{E}+\gamma\right) T_{0}}-\frac{\rho\left(\mu_{E}+\gamma\right) E^{2}}{\left(1+\alpha_{1} T_{0}\right)\left(\mu_{T}+\mu_{E}+\gamma\right) T_{0}} \\
& +\frac{\rho E}{\left(1+\alpha_{1} T_{0}\right) T_{0}}\left(T_{0}-T\right)-\frac{\mu_{I} \mu_{V}\left(\rho+\mu_{E}+\gamma\right)}{k \gamma} V .
\end{aligned}
$$


Hence,

$$
\begin{aligned}
D^{\alpha} L_{0}(t) \leq & -\left(\frac{1}{T}+\frac{\rho}{\left(\mu_{T}+\mu_{E}+\gamma\right) T_{0}}\right) \frac{\mu_{T}\left(T-T_{0}\right)^{2}}{1+\alpha_{1} T_{0}}-\frac{\rho\left(\mu_{E}+\gamma\right) E^{2}}{\left(1+\alpha_{1} T_{0}\right)\left(\mu_{T}+\mu_{E}+\gamma\right) T_{0}} \\
& -\frac{\rho\left(T-T_{0}\right)^{2} E}{\left(1+\alpha_{1} T_{0}\right) T T_{0}}+\frac{\mu_{I} \mu_{V}\left(\rho+\mu_{E}+\gamma\right)}{k \gamma}\left(R_{0}-1\right) V \\
& -\frac{\beta T_{0}\left(\alpha_{2}+\alpha_{3} T\right) V^{2}}{\left(1+\alpha_{1} T_{0}\right)\left(1+\alpha_{1} T+\alpha_{2} V+\alpha_{3} T V\right)} .
\end{aligned}
$$

Since $R_{0} \leq 1$, we have that $D^{\alpha} L_{0}(t) \leq 0$. Furthermore, $D^{\alpha} L_{0}(t)=0$ if and only if $T=T_{0}, E=0$ and $V=0$. From the last equation of (1), we get $I=0$. Consequently, the largest invariant set of $\left\{(T, E, I, V) \mid D^{\alpha} L_{0}(t)=0\right\}$ is the singleton $\left\{Q_{0}\right\}$. It follows from LaSalle's invariance principale [15] that the free equilibrium $Q_{0}$ is globally asymptotically stable when $R_{0}<1$.

By a simple computation, the characteristic equation at $Q_{0}$ is given by

$$
\left(\mu_{T}+\xi\right)\left(\xi^{3}+a_{1} \xi^{2}+a_{2} \xi+a_{3}\right)=0
$$

where

$$
\begin{aligned}
& a_{1}=\rho+\gamma+\mu_{E}+\mu_{I}+\mu_{V}, \\
& a_{2}=\mu_{I}\left(\rho+\gamma+\mu_{E}\right)+\mu_{V}\left(\rho+\gamma+\mu_{E}+\mu_{I}\right), \\
& a_{3}=\mu_{I} \mu_{V}\left(\rho+\gamma+\mu_{E}\right)\left(1-R_{0}\right) .
\end{aligned}
$$

Let

$$
P(\xi)=\xi^{3}+a_{1} \xi^{2}+a_{2} \xi+a_{3}
$$

We have $\lim _{\xi \rightarrow+\infty} P(\xi)=+\infty$ and $P(0)=\mu_{I} \mu_{V}\left(\rho+\gamma+\mu_{E}\right)\left(1-R_{0}\right)$. If $R_{0}>1$, then $P(0)<0$. So, there exists a $\xi_{0} \in(0,+\infty)$ such that $P\left(\xi_{0}\right)=0$, which implies that the characteristic equation at $Q_{0}$ has a positive root when $R_{0}>$ 1. Consequently $Q_{0}$ is unstable if $R_{0}>1$.

Theorem 3.2. The chronic infection equilibrium $Q_{1}$ is globally asymptotically stable if $R_{0}>1$ and

$$
R_{0} \leq 1+\frac{\left[\mu_{T} \mu_{I} \mu_{V}\left(\mu_{E}+\gamma\right)+\alpha_{2} \mu_{T} \lambda k \gamma\right]\left(\mu_{E}+\rho+\gamma\right)+\rho \alpha_{3} k \gamma \lambda^{2}}{\rho \mu_{I} \mu_{V}\left(\mu_{E}+\rho+\gamma\right)\left(\mu_{T}+\alpha_{1} \lambda\right)} .
$$


Proof. Consider the following Lyapunov functional

$$
\begin{aligned}
L_{1}(t)= & \frac{\left(1+\alpha_{2} V_{1}\right) T_{1}}{1+\alpha_{1} T_{1}+\alpha_{2} V_{1}+\alpha_{3} T_{1} V_{1}} \Phi\left(\frac{T}{T_{1}}\right) \\
& +\frac{\rho\left(1+\alpha_{2} V_{1}\right)}{2\left(1+\alpha_{1} T_{1}+\alpha_{2} V_{1}+\alpha_{3} T_{1} V_{1}\right)\left(\mu_{T}+\mu_{E}+\gamma\right) T_{1}}\left(T-T_{1}+E-E_{1}\right)^{2} \\
& +\frac{\rho+\mu_{E}+\gamma}{\gamma} I_{1} \Phi\left(\frac{I}{I_{1}}\right)+E_{1} \Phi\left(\frac{E}{E_{1}}\right)+\frac{\mu_{I}\left(\rho+\mu_{E}+\gamma\right)}{k \gamma} V_{1} \Phi\left(\frac{V}{V_{1}}\right) .
\end{aligned}
$$

The derivative of $L_{1}(t)$ along the positive solutions of (1) satisfies:

$$
\begin{aligned}
D^{\alpha} L_{1}(t) \leq & \left(1-\frac{f\left(T_{1}, V_{1}\right)}{f\left(T, V_{1}\right)}\right) D^{\alpha} T \\
& +\frac{\rho\left(1+\alpha_{2} V_{1}\right)\left(T-T_{1}+E-E_{1}\right)}{\left(1+\alpha_{1} T_{1}+\alpha_{2} V_{1}+\alpha_{3} T_{1} V_{1}\right)\left(\mu_{T}+\mu_{E}+\gamma\right) T_{1}}\left(D^{\alpha} T+D^{\alpha} E\right) \\
& +\frac{\rho+\mu_{E}+\gamma}{\gamma}\left(1-\frac{I_{1}}{I}\right) D^{\alpha} I+\left(1-\frac{E_{1}}{E}\right) D^{\alpha} E \\
& +\frac{\mu_{I}\left(\rho+\mu_{E}+\gamma\right)}{k \gamma}\left(1-\frac{V_{1}}{V}\right) D^{\alpha} V .
\end{aligned}
$$

By applying $\lambda=\mu_{T} T_{1}-f\left(T_{1}, V_{1}\right) V_{1}-\rho E_{1}=\mu_{T} T_{1}-\left(\gamma+\mu_{E}\right) E_{1}$, $\mu_{I}=\gamma \frac{E_{1}}{I_{1}}$ and $\mu_{V}=k \frac{I_{1}}{V_{1}}$, we get

$$
\begin{aligned}
D^{\alpha} L_{1}(t) \leq & \left(1-\frac{f\left(T_{1}, V_{1}\right)}{f\left(T, V_{1}\right)}\right) D^{\alpha} T \\
& +\frac{\rho\left(1+\alpha_{2} V_{1}\right)\left(T-T_{1}+E-E_{1}\right)}{\left(1+\alpha_{1} T_{1}+\alpha_{2} V_{1}+\alpha_{3} T_{1} V_{1}\right)()\left(\mu_{T}+\mu_{E}+\gamma\right) T_{1}}\left(D^{\alpha} T+D^{\alpha} E\right) \\
& +\frac{\rho+\mu_{E}+\gamma}{\gamma}\left(1-\frac{I_{1}}{I}\right) D^{\alpha} I+\left(1-\frac{E_{1}}{E}\right) D^{\alpha} E+\frac{\mu_{I}\left(\rho+\mu_{E}+\gamma\right)}{k \gamma}\left(1-\frac{V_{1}}{V}\right) D^{\alpha} V \\
\leq & \left(1-\frac{f\left(T_{1}, V_{1}\right)}{f\left(T, V_{1}\right)}\right)\left(-\mu_{T}\left(T-T_{1}\right)+\rho\left(E-E_{1}\right)-f(T, V) V+f\left(T_{1}, V_{1}\right) V_{1}\right) \\
& +\left(1-\frac{E_{1}}{E}\right)\left(f(T, V) V-\frac{f\left(T_{1}, V_{1}\right) V_{1}}{E_{1}} E\right)+\frac{\rho+\mu_{E}+\gamma}{\gamma}\left(1-\frac{I_{1}}{I}\right)\left(\gamma E-\frac{\gamma E_{1}}{I_{1}}\right) \\
& +\frac{\mu_{I}\left(\rho+\mu_{E}+\gamma\right)}{k \gamma}\left(1-\frac{V_{1}}{V}\right)\left(k I-\frac{k I_{1}}{V_{1}}\right) \\
& +\frac{\rho\left(1+\alpha_{2} V_{1}\right)\left[\left(T-T_{1}\right)+\left(E-E_{1}\right)\right]\left(-\mu_{T}\left(T-T_{1}\right)-\left(\mu_{E}+\gamma\right)\left(E-E_{1}\right)\right)}{T_{1}\left(1+\alpha_{1} T_{1}+\alpha_{2} V_{1}+\alpha_{3} T_{1} V_{1}\right)\left(\mu_{E}+\gamma+\mu_{T}\right)} .
\end{aligned}
$$


Thus,

$$
\begin{aligned}
D^{\alpha} L_{1}(t) \leq & \frac{-\mu_{T}\left(1+\alpha_{2} V_{1}\right)\left(T-T_{1}\right)^{2}}{T T_{1}\left(1+\alpha_{1} T_{1}+\alpha_{2} V_{1}+\alpha_{3} T_{1} V_{1}\right)}\left(\left(\mu_{T} T_{1}-\rho E_{1}\right)+\frac{\rho \mu_{T} T}{\mu_{E}+\gamma+\mu_{T}}+\rho E\right) \\
& -\frac{\rho\left(1+\alpha_{2} V_{1}\right)\left(\mu_{E}+\gamma\right)\left(E-E_{1}\right)^{2}}{T_{1}\left(1+\alpha_{1} T_{1}+\alpha_{2} V_{1}+\alpha_{3} T_{1} V_{1}\right)\left(\mu_{E}+\gamma+\mu_{T}\right)} \\
& +f\left(T_{1}, V_{1}\right) V_{1}\left(5-\frac{f\left(T_{1}, V_{1}\right)}{f\left(T, V_{1}\right)}-\frac{E I_{1}}{E_{1} I}-\frac{f(T, V)}{f\left(T_{1}, V_{1}\right)} \frac{E_{1} V}{E V_{1}}-\frac{I V_{1}}{I_{1} V}-\frac{f\left(T, V_{1}\right)}{f(T, V)}\right) \\
& -\frac{f\left(T_{1}, V_{1}\right)\left(1+\alpha_{1} T_{1}\right)\left(\alpha_{2}+\alpha_{3} T\right)\left(V-V_{1}\right)^{2}}{\left(1+\alpha_{1} T_{1}+\alpha_{2} V_{1}+\alpha_{3} T_{1} V_{1}\right)\left(1+\alpha_{1} T+\alpha_{2} V+\alpha_{3} T V\right)} .
\end{aligned}
$$

Since the arithmetic mean is greater than or equal to the geometric mean, it follows that

$$
5-\frac{f\left(T_{1}, V_{1}\right)}{f\left(T, V_{1}\right)}-\frac{E I_{1}}{E_{1} I}-\frac{f(T, V)}{f\left(T_{1}, V_{1}\right)} \frac{E_{1} V}{E V_{1}}-\frac{I V_{1}}{I_{1} V}-\frac{f\left(T, V_{1}\right)}{f(T, V)} \leq 0 .
$$

Therefore, $D^{\alpha} L_{1}(t) \leq 0$ if $\rho E_{1} \leqslant \mu_{T} T_{1}$. It is not hard to show that $\rho E_{1} \leqslant$ $\mu_{T} T_{1}$ is equivalent to (6). Further, $D^{\alpha} L_{1}(t)=0$ if and only if $E=E_{1}$, $V=V_{1}$ and $\frac{f\left(T_{1}, V_{1}\right)}{f\left(T, V_{1}\right)}=\frac{E I_{1}}{E_{1} I}=\frac{I V_{1}}{I_{1} V}$, which implies that $I=I_{1}$ and $T=$ $T_{1}$. By the LaSalle's invariance principale, we conclude that $Q_{1}$ is globally asymptotically stable.

Since

$$
\begin{aligned}
& \lim _{\rho \rightarrow 0} \frac{\left[\mu_{T} \mu_{I} \mu_{V}\left(\mu_{E}+\gamma\right)+\alpha_{2} \mu_{T} \lambda k \gamma\right]\left(\mu_{E}+\rho+\gamma\right)+\rho \alpha_{3} k \gamma \lambda^{2}}{\rho \mu_{I} \mu_{V}\left(\mu_{E}+\rho+\gamma\right)\left(\mu_{T}+\alpha_{1} \lambda\right)}=\infty, \\
& \lim _{\gamma \rightarrow \infty} \frac{\left[\mu_{T} \mu_{I} \mu_{V}\left(\mu_{E}+\gamma\right)+\alpha_{2} \mu_{T} \lambda k \gamma\right]\left(\mu_{E}+\rho+\gamma\right)+\rho \alpha_{3} k \gamma \lambda^{2}}{\rho \mu_{I} \mu_{V}\left(\mu_{E}+\rho+\gamma\right)\left(\mu_{T}+\alpha_{1} \lambda\right)}=\infty \text {, }
\end{aligned}
$$

we have the following result.

\section{Corollary 3.3.}

(i) The chronic infection equilibrium $Q_{1}$ is globally asymptotically stable if $R_{0}>1$ and $\rho$ is sufficiently small. In particular for $\rho=0$.

(ii) The chronic infection equilibrium $Q_{1}$ is globally asymptotically stable if $R_{0}>1$ and $\gamma$ is sufficiently large. 


\section{Numerical simulation}

In this section, we present some numerical simulations in order to illustrate our analytical results. The initial conditions of system (1) are $T(0)=800$ cells $\mathrm{mm}^{-3}, E(0)=100$ cells $\mathrm{mm}^{-3}, I(0)=24$ cells $\mathrm{mm}^{-3}$, and $V(0)=8000$ virions $\mathrm{mm}^{-3}$.

First, we choose $\Lambda=10, \mu_{T}=0.0139, \beta=0.000024, \alpha_{1}=0.1, \alpha_{2}=0.01$, $\alpha_{3}=0.00001, \rho=0.01, \gamma=1.1, \mu_{I}=0.29, \mu_{E}=0.0350, k=600$ and $\mu_{V}=3$. By calculation, we have $R_{0}=0.1568<1$. It follows from Theorem 2.2 that system (1) has a disease-free equilibrium $Q_{0}(719.4245,0,0,0)$. By Theorem 3.1, we see that $Q_{0}$ is globally asymptotically stable which means that the virus is cleared and the infection die out. Figure 1 illustrates this result.

Next, we choose $\beta=0.0005$ and we keep the other parameter values. We
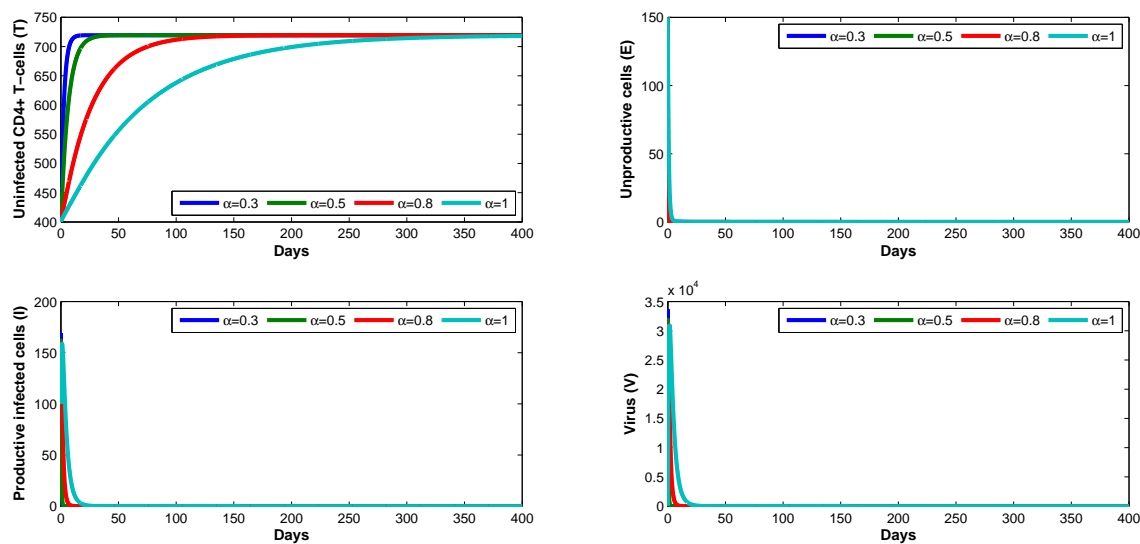

Figure 1: Stability of the disease-free equilibrium $Q_{0}$.

have $R_{0}=3.2673$ and

$$
1+\frac{\left[\mu_{T} \mu_{I} \mu_{V}\left(\mu_{E}+\gamma\right)+\alpha_{2} \mu_{T} \lambda k \gamma\right]\left(\mu_{E}+\rho+\gamma\right)+\rho \alpha_{3} k \gamma \lambda^{2}}{\rho \mu_{I} \mu_{V}\left(\mu_{E}+\rho+\gamma\right)\left(\mu_{T}+\alpha_{1} \lambda\right)}=107.2121
$$

Hence, the condition (6) is satisfied. From Theorem 2.2, the chronic infection equilibrium $Q_{1}(242.4,5.842,22.16,4232)$ is globally asymptotically stable, which means that the virus persists in the host and the infection becomes chronic. This result is confirmed by Figure 2 . 

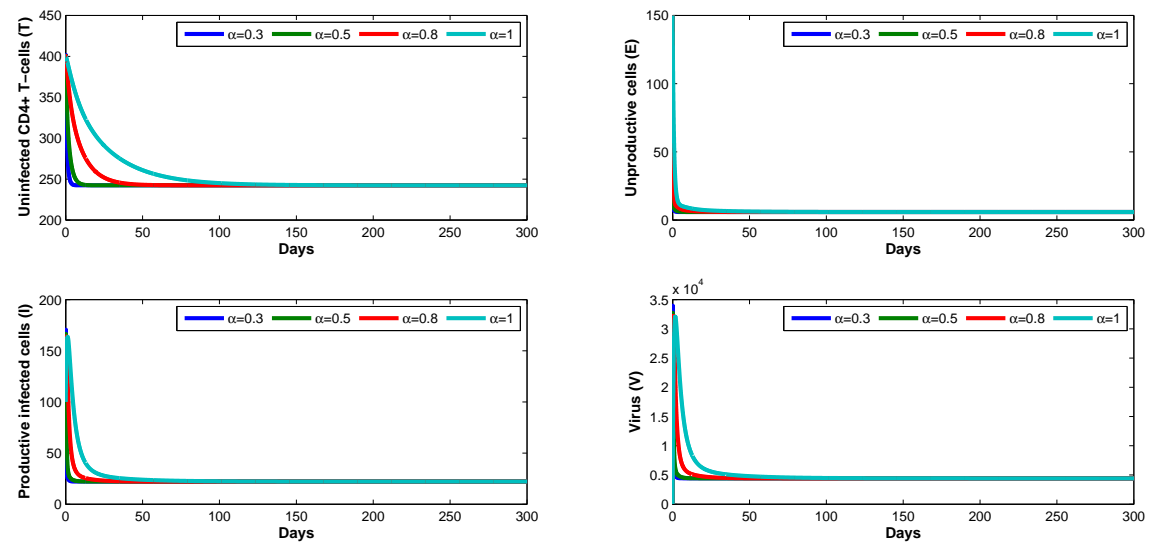

Figure 2: Stability of the chronic infection equilibrium $Q_{1}$.

Finally, we choose $\mu_{I}=0.27, \mu_{E}=0.0347, \beta=0.0084, \gamma=0.01, k=200$ and we keep the other parameter values. We have $R_{0}=3.7397$ and $1+\frac{\left[\mu_{T} \mu_{I} \mu_{V}\left(\mu_{E}+\gamma\right)+\alpha_{2} \mu_{T} \lambda k \gamma\right]\left(\mu_{E}+\rho+\gamma\right)+\rho \alpha_{3} k \gamma \lambda^{2}}{\rho \mu_{I} \mu_{V}\left(\mu_{E}+\rho+\gamma\right)\left(\mu_{T}+\alpha_{1} \lambda\right)}=1.4443$. Hence the dynamics of HIV infection converges to steady state $Q_{1}$, but the condition (6) is not satisfied. Therefore, the condition (6) is not necessary for the global stability of $Q_{1}$ (see Figure. 3).
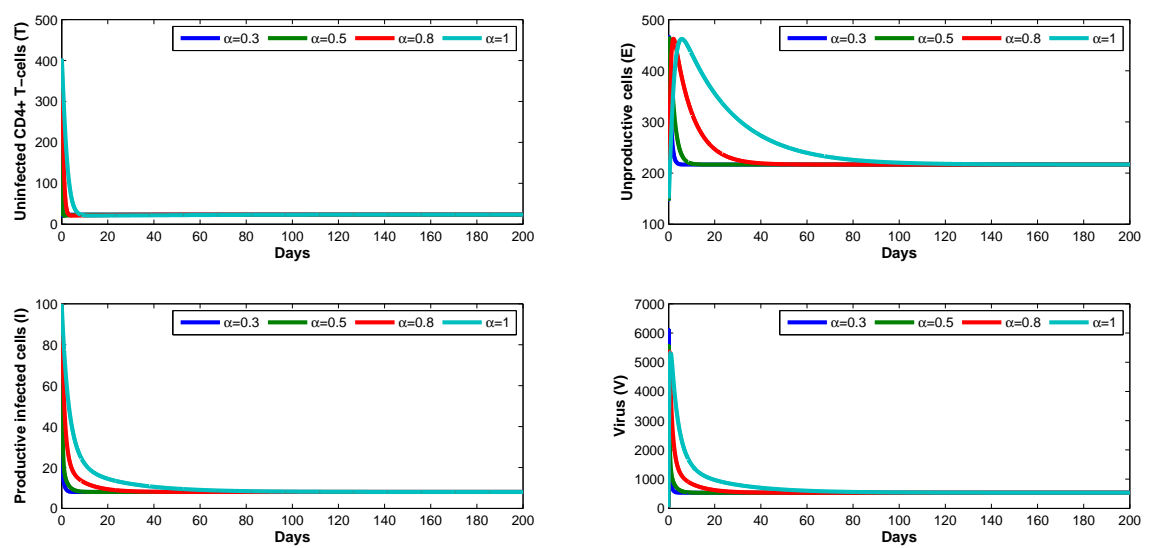

Figure 3: Dynamics of HIV infection with the condition (6) not satisfied. 


\section{Conclusion}

In this work, we have proposed a fractional-order model to describe the dynamics of HIV infection by taking into account the cure of infected cells in eclipse stage. We first proved that the proposed model is mathematically and virologically well-posed. In addition, we have proved that the diseasefree equilibrium $Q_{0}$ is globally asymptotically stable if the basic reproduction number $R_{0} \leq 1$, which mean that the HIV particles are eradicated. When $R_{0}>1, Q_{0}$ becomes unstable and there occurs the HIV infection equilibrium $Q_{1}$ which is globally asymptotically stable provided that the condition (6) is satisfied. In this case, the HIV particles persist in the host. Numerically, we see that the condition (6) is not necessary (see Figure 3). So, it will be interesting to prove it mathematically in future work. From our analytical and numerical results, we conclude that the fractional order has no effect on the asymptotic properties of the equilibria, but it may affect the time for arriving at these equilibria. In addition, the fractional-order models presented in $[1-4]$ are extended and improved.

\section{Acknowledgments}

The authors would like to thank the editor and the anonymous reviewer for their significant comments and suggestions that greatly improved the quality of the manuscript.

\section{References}

[1] A. A. M. Arafa, S. Z. Rida and M. Khalil, Fractional modeling dynamics of HIV and $\mathrm{CD} 4^{+}$T-cells during primary infection, Nonlinear Biomedical Physics 6 (1) (2012) 1-7.

[2] Y. Liu, J. Xiong, C. Hu and C. Wu, Stability Analysis for Fractional Differential Equations of an HIV Infection Model with Cure Rate, Proceedings of the IEEE International Conference on Information and $\mathrm{Au}-$ tomation, Ningbo, China, August (2016).

[3] S.M. Salman and A.M. Yousef, On a fractional-order model for HBV infection with cure of infected cells, Journal of the Egyptian Mathematical Society 25 (4) (2017) 445-451. 
[4] A. Boukhouima, K. Hattaf, N. Yousfi, Dynamics of a Fractional Order HIV Infection Model with Specific Functional Response and Cure Rate, International Journal of Differential Equations, (2017) 1-8.

[5] K. Hattaf, N. Yousfi, A. Tridane, Stability analysis of a virus dynamics model with general incidence rate and two delays, Applied Mathematics and Computation 221 (2013) 514-521.

[6] J. Adnani, K. Hattaf, N. Yousfi, Stability analysis of a stochastic SIR epidemic model with specific nonlinear incidence rate, Int. J. Stoch. Anal. (2013) Article ID 431257.

[7] K. Hattaf, N. Yousfi, Global dynamics of a delay reaction-diffusion model for viral infection with specific functional response, Computational and Applied Mathematics 34 (2015) 807-818.

[8] M. Maziane, E.M. Lotfi, K. Hattaf, N. Yousfi, Dynamics of class of HIV infection models with cure of infected cells in eclipse stage, Acta Biotheoretica 63 (2015) 363-380.

[9] M. Maziane, K. Hattaf, N. Yousfi, Global stability for a class of HIV infection models with cure of infected cells in eclipse stage and CTL immune response, Int. J. Dynam. Control 5 (2017) 1035-1045.

[10] J. R. Beddington, Mutual interference between parasites or predators and its effect on searching efficiency, J. Animal Ecol. 44 (1975) 331-340.

[11] D. L. DeAngelis, R. A. Goldstein, R. V. O'Neill, A model for trophic interaction, Ecology (1975) 881-892.

[12] P. H. Crowley, E. K. Martin, Functional responses and interference within and between year classes of a dragonfly population, J. North. Am. Benth. Soc. 8 (1989) 211-221.

[13] Z. Odibat and S. Momani, An algorithm for the numerical solution of differential equations of fractional order, J. Appl. Math. Inform. 26 (2008) $15-27$.

[14] Rong L, Gilchrist MA, Feng Z and Perelson AS, Modeling within-host HIV-1 dynamics and the evolution of drug resistance :trade-offs between viral enzyme function and drug susceptibility, J. Theoret, Biol 247 (2007) 804-818. 
[15] J. Huo, H. Zhao and L. Zhu, The effect of vaccines on backward bifurcation in a fractional order HIV model, Nonlinear Analysis: Real World Applications 26 (2015) 289-305.

[16] Z. Hu, W. Pang, F . Liao and W. Ma, Analysis of a CD4+ T cell viral infection model with a class of saturated infection rate, Discrete Continuous Dyn Syst Ser B 19 (2014) 735-745.

[17] K. S. Cole, Electric conductance of biological systems, Cold Spring Harbor Symposia on Quantitative Biology 1 (1933) 107-116.

[18] A. A. Arafa, S. Z. Rida, and M. Khalil, A fractional-order model of HIV infection: numerical solution and comparisons with data of patients, International Journal of Biomathematics, vol. 7 (2014) 1450036 1-11.

[19] C. V. De-Leon, Volterra-type Lyapunov functions for fractional-order epidemic systems, Communications in Nonlinear Science and Numerical Simulation 24 (2015) 75-85. 